\title{
Der steuerliche Umgang mit Verlusten: Reformoptionen für die Corona-Krise
}

\author{
Im Jahr 2020 werden viele Unternehmen durch die Corona-Pandemie Verluste erleiden. Das \\ deutsche Steuersystem behandelt Gewinne und Verluste asymmetrisch: Während Gewinne \\ sofort versteuert werden müssen, führen Verluste oft nicht zu direkten Steuererstattungen. \\ Dieser Beitrag diskutiert verschiedene Möglichkeiten, wie die steuerliche Behandlung \\ von Unternehmen in Verlustsituationen verbessert werden kann. Eine Mikrosimulation der \\ Reformoptionen zeigt, dass eine betragsmäßige Ausweitung der Verlustrücktragsmöglichkeiten \\ eine deutlich größere Wirkung entfaltet als ein längerer Rücktragszeitraum. Bessere \\ Verlustrücktragsmöglichkeiten setzen zudem starke Investitionsanreize.
}

\begin{abstract}
Unternehmen sind durch die Corona-Pandemie mit in dieser Form bisher nicht gekannten Einschränkungen konfrontiert. 725.000 der insgesamt gut 3 Mio. Unternehmen in Deutschland haben bis Mitte April 2020 Kurzarbeit beantragt. Viele Unternehmer befürchten im Jahr 2020 hohe Verluste. Bei der geltenden Rechtslage ist das Steuersystem aber nur bedingt geeignet, zur Überwindung kurzfristiger Liquiditätsengpässe beizutragen sowie Investitionsimpulse zu setzen.
\end{abstract}

\section{Geltendes Steuersystem: Steuersätze}

Die Gesamtsteuerbelastung der Unternehmen hängt in Deutschland von der Rechtsform ab. Bei Kapitalgesellschaften summiert sich die Steuerbelastung auf Gewinne auf Gesellschaftsebene (durch Körperschaftsteuer, Gewerbesteuer und Solidaritätszuschlag) auf durchschnittlich 31,6\% im Jahr 2018 (vgl. CTR 2018 in Abbildung 1). Deutschland liegt damit annähernd 10 Prozentpunkte oberhalb des EU-Durchschnitts von 22,2\%. Ein vergleichbares Bild zeigt sich bei der effektiven Durchschnittssteuerbelastung (vgl. EATR 2018 in Abbildung 1). ${ }^{1}$ Bei ausgeschütteten Gewinnen werden zudem die Kapitaleinkünfte der Anteilseigner besteuert. Deutschland zählt somit zu den Hochsteuerländern in Europa, weshalb sich in einer kurz vor Krisenbeginn durchgeführten Befragung deutscher Wirtschaftswissenschaftler die Mehrheit für eine Steuersatzsenkung ausgesprochen hat (Ökonomenpanel, 2020).

(C) Der/die Autor(en) 2020. Open Access: Dieser Artikel wird unter der Creative Commons Namensnennung 4.0 International Lizenz (https:// creativecommons.org/licenses/by/4.0/deed.de) veröffentlicht.

Open Access wird durch die ZBW - Leibniz-Informationszentrum Wirtschaft gefördert.

1 Die Zahlen entstammen einer Studie des Zentrums für Europäische Wirtschaftsforschung, vgl. Spengel et al. (2018).
Bei Personengesellschaften wird die Steuerbelastung wesentlich durch den persönlichen Einkommensteuersatz der Gesellschafter und durch Thesaurierungsentscheidungen bestimmt. Sie lässt sich aus diesem Grund weniger gut standardisieren. Unterliegen die Gesellschafter dem Spitzensteuersatz (wie für große, ertragsstarke Personengesellschaften angenommen werden kann), beträgt die Gesamtsteuerbelastung einer Personengesellschaft allerdings regelmäßig nahezu $50 \%$ und ist damit im internationalen Vergleich ebenfalls hoch.

\section{Geltendes Steuersystem: Verlustverrechnung}

In Krisen machen Unternehmen oft keine Gewinne, sondern Verluste. Somit entscheiden die Vorschriften zum Umgang mit Verlusten, wie stark Unternehmen in Krisensituationen durch Steuern belastet werden. ${ }^{2}$ Wie international üblich zeichnet sich die deutsche Unternehmensbesteuerung durch eine asymmetrische Behandlung von Gewinnen und Verlusten aus. Während Unternehmensge-

2 Auch weitere steuerrechtliche Regelungen, wie z. B. die Zinsschranke oder Hinzurechnungen bei der Gewerbesteuer, belasten Unternehmen in Verlustsituationen besonders.

Prof. Dr. Reinald Koch und Prof. Dr. Dominika Langenmayr sind Lehrstuhlinhaber an der Katholischen Universität (KU) Eichstätt-Ingolstadt und Institutssprecher des KU Research Institute for Taxation. Dominika Langenmayr ist zudem Research Fellow bei CESifo und der Research School of International Taxation der Universität Tübingen. 


\section{Abbildung 1}

\section{Steuerbelastung von Kapitalgesellschaften}

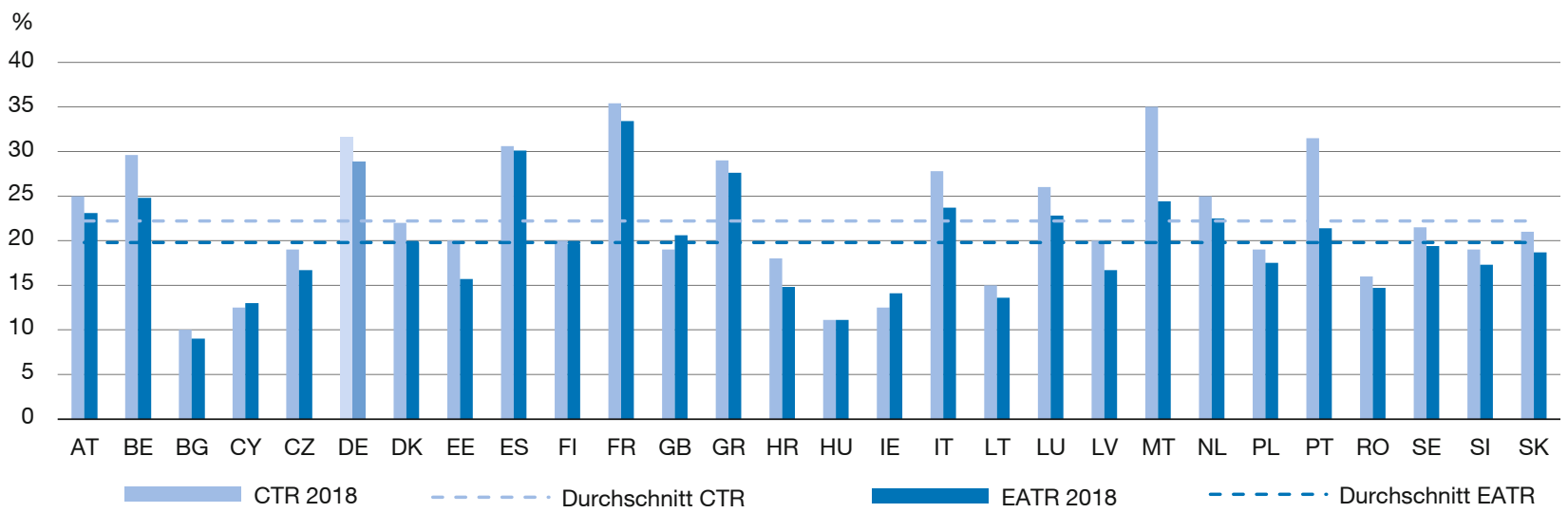

Anmerkungen: CTR = gesetzliche Steuerbelastung auf Gewinne, EATR = effektive Durchschnittssteuerbelastung. AT = Österreich, BE = Belgien, BG = Bulgarien, CY = Zypern, CZ = Tschechische Republik, DE = Deutschland, DK = Dänemark, EE = Estland, ES = Spanien, FI = Finnland, FR = Frankreich, $\mathrm{GB}=$ Großbritannien, GR = Griechenland, HR = Kroatien, HU = Ungarn, IE = Irland, IT = Italien, LT = Litauen, LU = Luxemburg, LV = Lettland, MT = Malta, $\mathrm{NL}=$ Niederlande, $\mathrm{PL}=$ Polen, $\mathrm{PT}=$ Portugal, $\mathrm{RO}=$ Rumänien, SE = Schweden, SI = Slowenien, SK = Slowakei.

Quelle: eigene Darstellung auf Basis der Ergebnisse in Spengel et al. (2018).

winne sofort besteuert werden, führen Verluste nicht zu einer unmittelbaren Steuererstattung im Sinne einer Negativsteuer. Verluste können vielmehr lediglich mit vergangenen oder zukünftigen Gewinnen verrechnet werden. Bei einem Verlustrücktrag wird der Verlust dabei mit Gewinnen aus der Vergangenheit verrechnet und die auf diese Gewinne gezahlte Steuer zurückerstattet. Dies geschieht im Rahmen der Steuererklärung des Verlustjahres, sodass die Steuererstattung erst im Jahr nach dem Verlust liquiditätswirksam wird. ${ }^{3}$ Der Verlustrücktrag in Deutschland ist auf ein Jahr und den Betrag von 1 Mio. Euro beschränkt. ${ }^{4}$

Wenn Verluste nicht oder nicht vollständig zurückgetragen werden können - weil es keinen Gewinn im Vorjahr gibt bzw. der Verlust diesen oder die Obergrenze von 1 Mio. Euro übersteigt -, wird der Verlust zeitlich unbeschränkt in Folgejahre vorgetragen und nach Maßgabe der Mindestbesteuerungsregelung mit zukünftigen Gewinnen verrechnet. Die Mindestbesteuerung besagt, dass Verlustvorträge nur bis zum Betrag von 1 Mio. Euro uneingeschränkt mit zukünftigen positiven Einkünften verrechnet werden können; darüber hinausgehende Verlustvorträge dürfen die positiven Einkünfte der Folgeperiode(n) nur zu $60 \%$ mindern. In Krisensituationen weist der Verlustvortrag konzeptionell den Nachteil auf, dass der steuerentlastende Effekt an

3 Der Sachverständigenrat zur Begutachtung der gesamtwirtschaftlichen Entwicklung (SVR für Wirtschaft) weist in seinem Sondergutachten richtigerweise daraufhin, dass die Entlastung somit allerdings nicht unmittelbar, sondern erst nach Abgabe der Steuererklärung zufließt. Vgl. SVR für Wirtschaft $(2020,77)$.

4 Die Möglichkeit zum Verlustrücktrag besteht bei der Einkommensteuer und Körperschaftsteuer, nicht aber bei der Gewerbesteuer. das Vorliegen zukünftiger Gewinne geknüpft ist und somit nur zeitlich verzögert und unter Unsicherheit einkalkuliert werden kann. Die Mindestbesteuerung führt zudem dazu, dass die entlastende Wirkung der Verlustverrechnung zu einem späteren Zeitpunkt eintritt.

Diese Regelungen sind im internationalen Vergleich

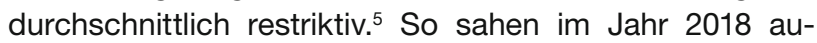
Ber Deutschland nur vier weitere EU-Staaten (Frankreich, Großbritannien, Irland und die Niederlande) die Möglichkeit zum Verlustrücktrag vor, davon allerdings mit Großbritannien, Irland und den Niederlanden drei Länder ohne betragsmäßige Begrenzung. ${ }^{6}$ Beim Verlustvortrag verwenden auch die meisten anderen EU-Länder eine betragsmäßige Begrenzung und/oder einen limitierenden Verlustvortragszeitraum. Lediglich vier Länder (Belgien, Irland, Malta und Schweden) erlauben einen unbegrenzten Verlustvortrag.

Die Angaben aus der letzten verfügbaren Körperschaftsteuerstatistik (für 2015 vgl. Statistisches Bundesamt, 2020) verdeutlichen, dass die deutschen Regelungen die Verlustverrechnung substanziell einschränken. Für 2015 sind in der Körperschaftsteuerstatistik insgesamt 1.175.306 unbeschränkt steuerpflichtige Kapitalgesellschaften ausgewiesen, von denen ein Drittel (388.999) im Jahr 2015 negative Einkünfte hat, die sich insgesamt auf knapp 60 Mrd. Euro belaufen. Drei Viertel dieser steuerlichen Verluste wurde

5 Die Angaben zum Steuerrecht der anderen EU-Staaten entstammen der Veröffentlichung des Bundesministeriums der Finanzen (2019).

6 In zeitlicher Hinsicht beschränken auch die anderen Länder den Verlustrücktrag auf ein Jahr; Großbritannien und Irland erweitern den Zeitraum im Fall der Betriebsaufgabe auf drei Jahre. 
von Unternehmen (Zahl: 6.092) erlitten, deren negative Einkünfte in diesem Jahr 1 Mio. Euro übersteigen und somit selbst bei ausreichend positiven steuerlichen Einkünften im Vorjahr - nicht vollständig rücktragfähig sind. Nach Abzug des Maximalrücktrags von 1 Mio. Euro betragen die nicht rücktragsfähigen Verluste mindestens 38,76 Mrd. Euro.

Wie stark schränkt die Mindestbesteuerung die Möglichkeiten zum Verlustvortrag ein? Etwa 3\% der Gesellschaften, die 2015 steuerliche Gewinne auswiesen (insgesamt: 786.307), haben Einkünfte, die den Sockelbetrag der Mindestbesteuerung von 1 Mio. Euro übersteigen. Die Einkünte dieser Unternehmen machen insgesamt (204 Mrd. Euro) mehr als $80 \%$ der Einkünfte aller Kapitalgesellschaften mit positiven Einkünften aus. Von diesen Unternehmen weisen mehr als $8 \%$ zum 31.12.2015 einen verbleibenden Verlustvortrag auf. Aufgrund der Mindestbesteuerung konnten sie 2015 nur 18 Mrd. Euro von insgesamt 130 Mrd. Euro Verlustvorträgen nutzen. Das zu versteuernde Einkommen (d. h. nach Verlustabzug) unterschritt daher den Gesamtbetrag der Einkünfte (vor Verlustabzug) um weniger als $9 \%{ }^{7}$

Für konzernverbundene Kapitalgesellschaften ergibt sich aus dem körperschaftsteuerlichen Trennungsprinzip eine zusätzliche Einschränkung der Verlustnutzung. Fallen in verschiedenen Tochtergesellschaften eines Konzerns zeitgleich Gewinne und Verluste an, so sind diese aufgrund der selbständigen Körperschaftsteuerpflicht der einzelnen Tochtergesellschaften grundsätzlich nicht verrechenbar. Etwas anderes gilt nur, wenn die Konzerngesellschaften gemeinsam eine körperschaftsteuerliche Organschaft bilden, für die eine derartige Verrechnung von Gewinnen und Verlusten zugelassen ist. Deutschland ermöglicht somit - wie auch 16 weitere EU-Länder - grundsätzlich eine konzerninterne Verlustverrechnung, begrenzt diese allerdings faktisch in zweierlei Hinsicht. Einerseits ist der Anwendungsbereich der Organschaft grundsätzlich auf inländische Tochterkapitalgesellschaften beschränkt, eine grenzüberschreitende Gruppenbesteuerung, wie beispielsweise in Österreich und Italien möglich, existiert in Deutschland nicht. Andererseits ist der Einbezug in eine körperschaftsteuerliche Organschaft für inländische Tochterkapitalgesellschaften an den Abschluss eines Ergebnisabführungsvertrags geknüpft, der faktisch die vollständige Übernahme der Haftung für Verluste der Tochterkapitalgesellschaft bedeutet. Oestreicher et al. $(2012,23)$ zeigen auf Basis von Daten für 2007 , dass aufgrund des Ergebnisabführungsvertrags die Organschaft nur für etwa $40 \%$ der Tochtergesellschaften in Anspruch genommen wird, welche die sonstigen Beteiligungsvoraussetzungen erfüllen. Auch nach der Körperschaftsteuerstatistik

7 Insgesamt betrugen die bis dato ungenutzten Verlustvorträge aller Gewinn- und Verlustunternehmen, die unter die betragsmäßigen Begrenzungen des Verlustvortrags und -rücktrags fallen, 388 Mrd. Euro.
2015 sind lediglich 3,2\% der Körperschaftsteuerpflichtigen (41.488 von 1.264.717) als Mutter- oder Tochtergesellschaft in eine körperschaftsteuerliche Organschaft einbezogen. ${ }^{8}$ Allerdings sind diese Konzerne überdurchschnittlich groß, sodass etwas mehr als ein Drittel aller körperschaftsteuerpflichtigen Einkünfte (265 Mrd. Euro von 708 Mrd. Euro) bei Kapitalgesellschaften in einer Organschaft anfällt.

\section{Reformvorschläge für die Unternehmensbesteuerung}

Im Verlauf der Corona-Krise wurde eine Reihe von Vorschlägen zur Reform der Unternehmensbesteuerung gemacht, die das Ziel haben, den Unternehmen kurz- und mittelfristig Liquidität zuzuführen, die Unternehmen grundsätzlich zu entlasten oder die Finanzierung von anderweitigen Hilfsmaßnahmen zu gewährleisten.

Zur Verbesserung der kurzfristigen Liquidität wurde bereits umgesetzt, dass Unternehmen unter gewissen Voraussetzungen Einkommen- und Körperschaftsteuervorauszahlungen (und gegebenenfalls auch Gewerbesteuervorauszahlungen) herabsetzen können sowie Einkommen-, Körperschaft- und Umsatzsteuerschulden gestundet werden können. ${ }^{9}$ Weitergehende Vorschläge sehen übereinstimmend vor, die Möglichkeiten zur steuerlichen Verlustverrechnung auszuweiten. Dabei wird eine Ausweitung des Verlustrücktrags in zeitlicher und/oder betragsmäßiger Hinsicht empfohlen (Bundesverband der Deutschen Industrie [BDI], 2020; Eberhartinger und Petutschnig, o.D.; Koch und Langenmayr, 2020; SVR für Wirtschaft, 2020; Gassen et al., 2020; Giese et al., 2020). Teilweise wird gefordert, die entlastende Wirkung des Verlustrücktrags durch eine Erstattung der im Vorjahr gezahlten Steuern unmittelbar, d.h. noch 2020, herbeizuführen (Eberhartinger und Petutschnig, o.D.; Gassen et al., 2020). Zudem könnten durch die Bildung einer unversteuerten Rücklage zu erwartende Verluste bereits bei der Ermittlung des steuerlichen Gewinns für das Jahr 2019 gewinnmindernd berücksichtigt werden (Eberhartinger und Langenmayr, 2020; Eberhartinger und Petutschnig, o.D.).

Mit Blick auf den Verlustvortrag wird vorgeschlagen, die Mindestbesteuerung für Verluste aus der Krise auszusetzen bzw. die Grenzen der Mindestbesteuerung anzupassen (BDI, 2020 bzw. SVR für Wirtschaft, 2020). Da auch die Zinsschrankenregelung krisenverschärfend wirken kann, regen der BDI (2020) und Koch und Langenmayr (2020) ferner ein temporäres Aussetzen der Zinsschrankenregelung an. Uneinheitlich wird eine Verbesserung der Abschreibungsbedingungen gesehen. Während der BDI eine temporäre

8 Im Vergleich zur Körperschaftsteuerstatistik 2007 (2,7\%) ist dieser Anteil nun zwar etwas gestiegen, die grundsätzliche Aussage aus Oestreicher et al. (2012) sollte allerdings weiterhin Bestand haben.

9 Vgl. BMF-Schreiben vom 19.3.2020, IV A 3 -S 0336/19/10007:002. 
Wiedereinführung der degressiven Abschreibung wie auch bereits in der Finanzkrise befürwortet, weist der SVR für Wirtschaft $(2020,77)$ in seinem Gutachten darauf hin, dass von verbesserten Abschreibungsmöglichkeiten "lediglich eine eingeschränkte und wenig zielgerichtete Wirkung" ausginge.

Kontrovers diskutiert wird die zukünftige Entwicklung der Steuersätze mit Blick auf die Corona-Krise. Während der BDI ebenso wie Vertreter von CDU/CSU und FDP sich für eine Senkung der Steuersätze zur Stärkung der Unternehmen und als Investitionsanreiz aussprechen, wird dagegen angeführt, dass eine Anhebung der Steuersätze zur Finanzierung diverser Hilfsmaßnahmen und zur Sicherung öffentlicher Finanzen notwendig sei. In diesem Sinne hat sich beispielsweise der Städte- und Gemeindebund für die Einführung eines „Corona-Soli“ ausgesprochen.

\section{Simulation der verschiedenen Reformvorschläge}

Welche Entlastungswirkungen für Unternehmen bzw. welche Aufkommenseinbußen wären voraussichtlich mit den oben genannten Reformvorschlägen verbunden? Und welche Unternehmen würden von welchen Maßnahmen besonders profitieren? Um für die Beantwortung dieser Fragen erste Hinweise zu geben, ermitteln wir mithilfe einer Mikrosimulation die Auswirkungen verschiedener Reformmöglichkeiten bei der Verlustverrechnung auf die Körperschaftsteuerzahlungen von Kapitalgesellschaften sowie die korrespondierenden Erstattungsansprüche aus Verlustrückträgen. Die Berechnungen basieren auf einem Paneldatensatz mit Finanzinformationen zu 30.913 deutschen Kapitalgesellschaften aus der ORBIS-Datenbank von Bureau van Dijk für den Zeitraum von 2008 bis (maximal) 2019. Ausgangspunkt der Berechnungen bildet das Ergebnis vor Steuern, das auf Basis eines AR(1)-Prognosemodells bis 2022 fortgeschrieben wird. ${ }^{10}$ Wir bilden dabei den exogenen Schock der Corona-Krise ab, indem wir bei der Schätzung der AR(1)Parameter eine Variable berücksichtigen, welche die Veränderung des Bruttoinlandsprodukts (BIP) im laufenden Jahr widerspiegelt. Für 2020 und 2021 verwenden wir hierzu die Konjunkturprognose des SVR für Wirtschaft.11

Abbildung 2 zeigt die kumulierten Ergebnisse der Unternehmen in unserem Datensatz. Die Werte für die Krisenjahre 2020 und 2021 verdeutlichen, dass die verwendete Metho-

10 Die Methodik basiert auf der Vorgehensweise des von Graham und Kim entwickelten Modells zur Simulation marginaler Steuersätze, vgl. Graham (1996, 41-74) und Graham und Kim (2009). Eine vergleichbare Vorgehensweise wählt auch das an der Georg-August-Universität Göttingen entwickelte Mikrosimulationsmodell ASSERT, vgl. Oestreicher et al. (2013).

11 Für 2022 wird das durchschnittliche BIP-Wachstum von 2008 bis 2019 unterstellt.
Abbildung 2

Entwicklung der kumulierten Gewinne und Verluste (vor Steuern)

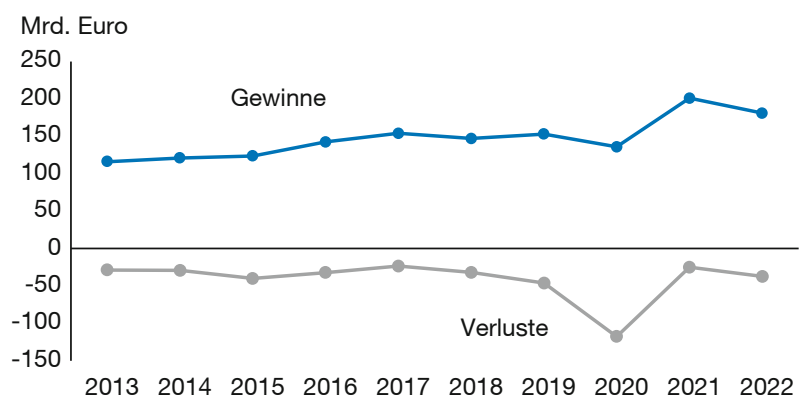

Anmerkung: Die Gewinne und Verluste basieren in den Jahren 2013 bis 2019 auf den in ORBIS direkt berichteten Daten, während die Werte für 2020 bis 2022 simuliert sind. Soweit Angaben in ORBIS für die Jahre 2018 oder 2019 fehlen, sind auch diese simuliert.

Quelle: eigene Berechnungen.

dik die Krise sinnvoll abbildet: Die im simulierten Szenario unterstellte Entwicklung des BIP (2020: Rückgang um 5,4\%; 2021: Anstieg um 4,9\%) führt zu einem deutlichen Anstieg der Verluste 2020 und einer gegenläufigen Bewegung $2021 .^{12}$

Durch einen Vergleich mit den drei zuletzt veröffentlichten Körperschaftsteuerstatistiken (2013 bis 2015) lässt sich die Repräsentativität der verwendeten Daten beurteilen. In ORBIS sind durchschnittlich $52 \%$ der Gewinne und $57 \%$ der Verluste, die in der Körperschaftsteuerstatistik ausgewiesen sind, abgebildet. Die Daten repräsentieren somit mehr als die Hälfte der Gewinne und Verluste deutscher Kapitalgesellschaften und sind hinsichtlich der Verteilung von positiven und negativen Ergebnissen weitgehend repräsentativ. Da die enthaltenen 30.913 Unternehmen lediglich etwa 2,7 \% aller deutschen Körperschaftsteuerpflichtigen entsprechen, sind große Unternehmen deutlich überrepräsentiert.

Im Rahmen der Mikrosimulation ermitteln wir auf Basis der prognostizierten Ergebnisse die zu zahlende Körperschaftsteuer und den Solidaritätszuschlag. Wir berücksichtigen dabei die Regelung zum Verlustrücktrag und -vortrag nach geltendem Recht und einer Reihe von Reformvorschlägen. Die zum 1.1.2020 bestehenden steuerlichen Verlustvorträge werden dabei unter Verwendung der gesamten verfügbaren Datenhistorie unternehmensindividuell simuliert.

12 Dieses Szenario wird im Sondergutachten des SVR für Wirtschaft als erstes Risikoszenario (ausgeprägtes $\mathrm{V}$ ) bezeichnet. 
Die Tabelle 1 zeigt die Ergebnisse der Mikrosimulation für die ORBIS-Stichprobe bei geltendem Recht und verschiedenen Reformoptionen. Bezogen auf alle Unternehmen in der Stichprobe zeigt die betragsmäßige Ausweitung des Verlustrücktrags die größte Wirkung. Selbst wenn der Verlustrücktrag auf ein Jahr begrenzt bleibt und nur die Obergrenze von 1 Mio. Euro aufgehoben wird, ist die Entlastungswirkung (-4,2\%) größer als beispielsweise die Anhebung des Sockelbetrags bei der Mindestbesteuerung auf 5 Mio. Euro $(-1,2 \%){ }^{13}$ Demgegenüber entfaltet die zeitliche Ausdehnung des Verlustrücktrags ohne gleichzeitige Abschaffung der Obergrenze keine nennenswerte Wirkung. Insgesamt zeigt sich allerdings, dass auch bei sehr großzügiger Ausgestaltung der Rücktragsregelung (Verlustrücktrag 5 Jahre, unbegrenzt) lediglich 50,72 Mrd. Euro 2020 zurückgetragen werden können. Dieses ist immer noch weniger als die Hälfte der 2020 eingetretenen Verluste. Diese Ergebnisse verdeutlichen ferner, dass eine sofortige Steuererstattung als vorweggenommener Verlustrücktrag nur sinnvoll ist, wenn gleichzeitig die Rücktragsmöglichkeiten verbessert werden. ${ }^{14}$ Die letzte Spalte in Tabelle 1 verdeutlicht, wie die Veränderungen bei der Verlustverrechnung bei gleichzeitiger Senkung der Steuersätze (hier: auf 10\%) ab 2020 wirken. Da die Verluste größtenteils noch zum höheren alten Steuersatz erstattet werden, ist die Entlastung größer, als es die Steuersatzsenkung um ungefähr $33 \%$ auf den ersten Blick vermuten lassen würde. Aus diesem Grund ist insbesondere eine Ausweitung des Verlustrücktrags in diesem Szenario für die Unternehmen besonders vorteilhaft.

Inwiefern hängt die Entlastungswirkung der verschiedenen Reformoptionen von der Unternehmensgröße ab? Zur Beantwortung dieser Frage gliedern wir in Tabelle 2 die Auswirkungen der Reformszenarien in drei Größenklassen auf. Die Tabelle 2 zeigt die Ergebnisse der Mikrosimulation für die ORBIS-Stichprobe bezüglich der Steuerzahlung nach Unternehmensgröße für die verschiedenen Reformoptionen. Wenn die betragsmäßigen Beschränkungen des Verlustrücktrags abgeschafft würden, hätte dies keine große Wirkung bei kleinen Unternehmen, während größere Unternehmen deutlich profitieren. Möchte der Gesetzgeber Unternehmen aus unterschiedlichen Größenklassen möglichst gleichmäßig entlasten, ist eine Erhöhung des Sockelbetrags der Mindestbesteuerung auf 5 Mio. Euro eine mögliche Reformoption, die allerdings bei allen Unternehmen

13 Die Kosten für den Staat sind dabei geringer als die prozentualen Mindereinnahmen suggerieren, da die entsprechenden Verluste nach geltendem Recht zumindest teilweise zukünftig (nach dem Simulationszeitraum) im Rahmen des Verlustvortrags genutzt werden würden.

14 Die Berechnungen beziehen sich auf die Stichprobe aus ORBIS, die überproportional viele große Unternehmen enthält. Eine Hochrechnung anhand der Größenklassen in der Körperschaftsteuerstatistik zeigt, dass sich die Ergebnisse dadurch nur geringfügig (um maximal 1 Prozentpunkt) verändern, d.h. gesamtwirtschaftlich sind ähnliche prozentuale Änderungen des Steueraufkommens zu erwarten.
Tabelle 1

Auswirkungen der verschiedenen Reformoptionen

\begin{tabular}{|c|c|c|c|c|c|}
\hline & 2020 & 2021 & 2022 & $\begin{array}{l}\text { Ge- } \\
\text { samt }^{2}\end{array}$ & $\begin{array}{l}\text { Steuer- } \\
\text { satzän- } \\
\text { derung }^{3}\end{array}$ \\
\hline & \multicolumn{3}{|c|}{$\begin{array}{l}\text { Steuerbarer Gewinn in Mrd. Euro' } \\
\text { Verlustrücktrag in Mrd. Euro }\end{array}$} & \multicolumn{2}{|c|}{$\begin{array}{l}\text { Änderung der } \\
\text { Steuerzahlung } \\
\text { in \% }\end{array}$} \\
\hline $\begin{array}{l}\text { Geltendes } \\
\text { Recht }\end{array}$ & $\begin{array}{l}128,9 \\
-2,95\end{array}$ & $\begin{array}{r}168,99 \\
-0,61\end{array}$ & $\begin{array}{r}164,78 \\
-1,26\end{array}$ & - & $-37,10$ \\
\hline $\begin{array}{l}\text { Verlustrücktrag } \\
1 \text { Jahr, Höhe } \\
\text { unbegrenzt }\end{array}$ & $\begin{array}{r}128,9 \\
-14,03\end{array}$ & $\begin{array}{r}172,72 \\
-3,28\end{array}$ & $\begin{array}{r}166,68 \\
-12,55\end{array}$ & $-4,20$ & $-40,60$ \\
\hline $\begin{array}{l}\text { Verlustrücktrag } \\
3 \text { Jahre }\end{array}$ & $\begin{array}{l}128,9 \\
-4,84\end{array}$ & $\begin{array}{r}169,47 \\
-1,10\end{array}$ & $\begin{array}{r}164,99 \\
-1,55\end{array}$ & $-0,40$ & $-37,50$ \\
\hline $\begin{array}{l}\text { Verlustrücktrag } \\
3 \text { Jahre, Höhe } \\
\text { unbegrenzt }\end{array}$ & $\begin{array}{r}128,9 \\
-38,05\end{array}$ & $\begin{array}{r}177,54 \\
-6,06\end{array}$ & $\begin{array}{r}169,36 \\
-14,46\end{array}$ & $-8,90$ & $-45,50$ \\
\hline $\begin{array}{l}\text { Verlustrücktrag } \\
5 \text { Jahre }\end{array}$ & $\begin{array}{l}128,9 \\
-5,59\end{array}$ & $\begin{array}{r}169,56 \\
-1,37\end{array}$ & $\begin{array}{r}165,04 \\
-1,89\end{array}$ & $-0,70$ & $-37,70$ \\
\hline $\begin{array}{l}\text { Verlustrücktrag } \\
5 \text { Jahre, Höhe } \\
\text { unbegrenzt }\end{array}$ & $\begin{array}{r}128,9 \\
-50,72\end{array}$ & $\begin{array}{r}180,36 \\
-8,25\end{array}$ & $\begin{array}{l}170,14 \\
-16,79\end{array}$ & $-11,80$ & $-48,40$ \\
\hline $\begin{array}{l}\text { Verlustvortrag } \\
\text { maximal } \\
5 \text { Mio. Euro }\end{array}$ & $\begin{array}{r}128,02 \\
-2,95\end{array}$ & $\begin{array}{r}165,09 \\
-0,53\end{array}$ & $\begin{array}{r}163,79 \\
-0,94\end{array}$ & $-1,20$ & $-37,80$ \\
\hline $\begin{array}{l}\text { Verlustvortrag } \\
\text { ohne Mindest- } \\
\text { besteuerung }\end{array}$ & $\begin{array}{r}126,15 \\
-2,96\end{array}$ & $\begin{array}{r}155,07 \\
-0,47\end{array}$ & $\begin{array}{r}161,82 \\
-0,68\end{array}$ & $-4,10$ & $-39,70$ \\
\hline
\end{tabular}

${ }^{1}$ Für 2020 bis 2022 sind die kumulierten steuerlichen Einkünfte (obere Zeile) sowie die kumulierten genutzten Verlustrückträge (untere Zeile) angegeben. ${ }^{2}$ Kumulierte Steuerzahlung beim geltenden Körperschaftsteuersatz von $15 \%$ (plus $0,825 \%$ Solidaritätszuschlag) sowie die Aufkommensveränderung bei den verschiedenen Reformoptionen gegenüber den simulierten Steuereinnahmen bei geltendem Recht (72,46 Mrd. Euro). ${ }^{3}$ Aufkommensentwicklung bei einer Senkung des Körperschaftsteuersatzes auf $10 \%$ und Abschaffung des Solidaritätszuschlags relativ zu den simulierten Steuereinnahmen bei geltendem Recht (72,46 Mrd. Euro).

Quelle: eigene Berechnungen.

nur eine geringe Entlastungswirkung hat. Zu beachten ist zudem, dass Verlustvorträge erst später liquiditätswirksam werden und somit weniger Investitionsimpulse direkt nach der Corona-Krise geben können.

\section{Anreizwirkungen der verschiedenen Reformvorschläge}

Bei der Berechnung der Aufkommenseffekte haben wir angenommen, dass die steuerlichen Änderungen keine Auswirkungen auf das Verhalten von Unternehmen haben. Dies ist unrealistisch: Es wäre ja gerade der Sinn solcher Reformen, Unternehmen zu mehr Investitionen anzuregen und einen schnelleren Neustart der Wirtschaft zu fördern. Eine Vielzahl von Studien hat analysiert, wie sich Steuersätze auf Unternehmensaktivitäten, insbesondere Investitionen, auswirken. Der Grundgedanke ist, dass Steuern Gewinne verrin- 
Tabelle 2

Auswirkungen der Reformen auf die Steuerzahlung nach Unternehmensgröße

\begin{tabular}{|c|c|c|c|}
\hline & $\begin{array}{c}\text { Größenklasse } 1 \\
\text { Bilanzsumme } \\
<6 \text { Mio. Euro } \\
\text { in } \%\end{array}$ & $\begin{array}{c}\text { Größenklasse } 2 \\
\text { Bilanzsumme > } \\
6 \text { Mio. }<20 \text { Mio. } \\
\text { Euro in \% }\end{array}$ & $\begin{array}{c}\text { Größenklasse } 3 \\
\text { Bilanzsumme } \\
>20 \text { Mio. Euro } \\
\text { in } \%\end{array}$ \\
\hline $\begin{array}{l}\text { Verlustrücktrag } \\
1 \text { Jahr, unbegrenzt }\end{array}$ & $-0,30$ & $-0,60$ & $-4,70$ \\
\hline $\begin{array}{l}\text { Verlustrücktrag } \\
3 \text { Jahre }\end{array}$ & $-2,10$ & $-1,30$ & $-0,30$ \\
\hline $\begin{array}{l}\text { Verlustrücktrag } \\
3 \text { Jahre, unbegrenzt }\end{array}$ & $-3,30$ & $-3,00$ & $-9,70$ \\
\hline $\begin{array}{l}\text { Verlustrücktrag } \\
5 \text { Jahre }\end{array}$ & $-3,70$ & $-2,30$ & $-0,50$ \\
\hline $\begin{array}{l}\text { Verlustrücktrag } \\
5 \text { Jahre, unbegrenzt }\end{array}$ & $-5,30$ & $-4,70$ & $-12,80$ \\
\hline $\begin{array}{l}\text { Verlustvortrag } \\
5 \text { Mio. Euro }\end{array}$ & $-0,20$ & $-0,40$ & $-0,50$ \\
\hline $\begin{array}{l}\text { Verlustvortrag } \\
\text { ohne Mindestbe- } \\
\text { steuerung }\end{array}$ & $-0,20$ & $-0,50$ & $-4,20$ \\
\hline
\end{tabular}

Anmerkung: Die Prozentwerte beziehen sich auf die kumulierte Steuerzahlung für die Jahre 2020 bis 2022 relativ zum geltenden Recht.

Quelle: eigene Berechnungen.

gern, und sich daher Investitionen bei höheren Steuersätzen weniger lohnen. ${ }^{15}$ Empirisch ist dies gut belegt: Djankov et al. (2010) vergleichen die Steuerbelastung für ein standardisiertes Unternehmen zwischen Ländern und zeigen, dass eine um 10 Prozentpunkte niedrigere effektive Gewinnsteuerbelastung zu einem 2 Prozentpunkte höheren gesamtwirtschaftlichen Investitionsniveau relativ zum BIP führt (durchschnittliche Investitionen/BIP: $21 \%$ ). Dies gilt auch in Deutschland: Dobbins und Jacob (2016) untersuchen die Unternehmensteuerreform 2008. Demnach führte die um 10 Prozentpunkte niedrigere Steuerbelastung zu 5,9\% mehr Investitionen. Dies entspricht einem Anstieg des Anlagevermögens von ca. 3 Mio. Euro bei einem durchschnittlichen Unternehmen. Die zusätzlichen Investitionen entsprechen ungefähr dem Umfang der Mindereinnahmen bei der Körperschaftsteuer, d.h. 1 Euro Aufkommensverlust führte zu 1 Euro zusätzlichen Investitionen. Studien aus anderen Ländern kommen zu sehr ähnlichen Ergebnissen (vgl. beispielsweise Ohrn, 2018).

\section{Ergebnisse für Unternehmen in Verlustsituationen}

Wenn Unternehmen ungenutzte Verlustvorträge haben, mindern diese die zu erwartende Steuerbelastung. Sind die

15 Theoretisch ist der Effekt von Steuern auf Investitionen nicht so eindeutig: Werden Investitionsentscheidungen durch die Bildung von Kapitalwerten getroffen, beeinflussen Steuern nicht nur die Erträge, sondern auch den Diskontierungszins. erwarteten Erträge geringer als der Sockelbetrag der Mindestbesteuerung, werden die Erträge quasi nicht besteuert - das Investitionsverhalten ist dann unabhängig vom Steuersatz. Für größere Unternehmen, bei denen die Mindestbesteuerung greift, verringern die vorgetragenen Verluste die effektive Steuerbelastung noch zu $60 \%$. Möchte man Investitionen anregen, wäre es zielführend, die Mindestbesteuerung zumindest temporär auszusetzen oder den Sockelbetrag zu erhöhen. Steuersatzsenkungen sind insbesondere dann hilfreich, wenn Unternehmen die Verluste bereits durch Verlustrückträge nutzen konnten oder um der Wirtschaft nach der Krise einen zusätzlichen Impuls für langfristige Investitionen zu geben.

Die Möglichkeit, Verluste zu verrechnen, regt also Investitionen an, wenn die Unternehmen bereits Verluste haben, da sie die negative Wirkung des Steuersatzes abschwächt bzw. aufhebt. Zudem setzt die Existenz von Verlustverrechnungsregelungen auch einen Anreiz, insbesondere risikoreiche Investitionen (beispielsweise in Forschung und Entwicklung) zu tätigen. Durch die Möglichkeit der Verlustverrechnung trägt der Staat einen Teil des Investitionsrisikos, er beteiligt sich über das Steuersystem an Gewinnen und Verlusten. Langenmayr und Lester (2018) belegen diesen Effekt empirisch. Sie zeigen, dass Unternehmen 11,6\% mehr riskante Investitionen tätigen, wenn der Zeitraum, in dem ein Verlustrücktrag möglich ist, um ein Jahr erweitert wird. Dieser Effekt ist dabei in Ländern mit höheren Steuersätzen größer, da hier die „Versicherungsfunktion“, die der Staat durch die steuerliche Beteiligung an Verlusten übernimmt, eine größere Rolle spielt. Ein ähnlicher Effekt ist auch bei einer Aufhebung der Obergrenze von 1 Mio. Euro zu erwarten, auch wenn dies in der Studie nicht explizit untersucht wird.

Zusammenfassend zeigt sich somit, dass - gerade in der Krise, aber auch langfristig - die steuerlichen Regelungen zur Verlustverrechnung von enormer Bedeutung für die Investitionstätigkeit sind. ${ }^{16}$

16 Dies bestätigen auch Analysen zu steuerlichen Maßnahmen in Krisen in der Vergangenheit: Zwick und Mahon (2017) analysieren die Auswirkungen von Sonderabschreibungen, die in den USA in den letzten beiden Wirtschaftskrisen temporär eingeführt wurden (2001 bis 2004 und 2008 bis 2010). In der letzten Krise erlaubten die USA degressive Abschreibungen mit Abschreibungssätzen von $50 \%$ und mehr, was zu $17 \%$ höheren Investitionen in Wirtschaftsgüter, für die diese Abschreibungen möglich waren, führte. Kleinere Unternehmen reagierten dabei deutlich stärker auf diese Anreize als größere Unternehmen. Somit können auch in Deutschland, wo die Wirtschaft deutlich mehr durch mittelständische Unternehmen geprägt ist, substanzielle Investitionsanreize erwartet werden. Zwick und Mahon zeigen dabei allerdings auch, dass Unternehmen nur dann zusätzliche Investitionen durchführen, wenn die Sonderabschreibungen zeitnahe Liquiditätseffekte haben. Direkt nach der Krise wird dies für viele Unternehmen nur der Fall sein, wenn sie Verlustrückträge nutzen können. Damit ist auch diese Maßnahme nur zielführend, wenn gleichzeitig die Möglichkeiten zum Verlustrücktrag verbessert werden. 


\section{Empfehlungen}

So, wie Unternehmen mit einem Teil ihrer Gewinne durch ihre Steuerzahlung zur Finanzierung des Staats beitragen, sollten auch Verluste die Steuerzahlungen der Unternehmen mindern. Die Regelungen im geltenden Recht sind dafür unzureichend: Nur gut $10 \%$ der simulierten Verluste im Jahr 2020 werden nach geltendem Recht durch einen Verlustrücktrag zeitnah zu Steuererstattungen führen. Erholt sich die Wirtschaft langsam, werden viele Unternehmen die jetzt entstehenden Verluste erst in vielen Jahren nutzen können - wenn das Unternehmen dann noch existiert.

Unsere Analysen zeigen, dass insbesondere die betragsmäßigen Begrenzungen eine effektive Verlustverrechnung verhindern. Selbst wenn der Verlustrücktrag auf ein Jahr begrenzt bleibt und nur die Obergrenze von 1 Mio. Euro aufgehoben wird, werden Unternehmen mit Verlusten deutlich entlastet (Steuermindereinnahmen von 4,2\% im Vergleich zum geltenden Recht). Ein weiterer Vorteil einer solchen Reform wäre, dass sie relativ zielgenau Unternehmen hilft, die in der jüngsten Vergangenheit profitabel waren, aber von der Krise stark betroffen sind.

Allerdings würde eine reine Aufhebung der Obergrenze kleinere Unternehmen nicht nennenswert entlasten. Diese könnten durch eine Verlängerung des Rücktragszeitraums auf drei Jahre entlastet werden. Eine solche Reform wäre aber auch mit höheren Kosten für den Staat verbunden (Steuermindereinnahmen von 8,9\%). Auch eine Kombination (unbegrenzter Rücktrag in das Vorjahr, Rücktrag in die zwei Jahre zuvor begrenzt auf z.B. 1 Mio. Euro) wäre denkbar. Unabhängig davon, wie die Verbesserung der Verlustverrechnung ausgestaltet wird: Eine Reform in diese Richtung würde den Unternehmen den Neustart nach der Krise erleichtern und zeitnah Investitionsanreize setzen.

\section{Literatur}

Bundesministerium der Finanzen (2019), Die wichtigsten Steuern im internationalen Vergleich 2018, https://www.bundesfinanzministerium.de/ Content/DE/Downloads/Broschueren_Bestellservice/2019-08-08-diewichtigsten-steuern-im-internationalen-vergleich-2018-ausgabe-2019. pdf?__blob=publicationFile\&v=9 (23. April 2020).
Bundesverband der Deutschen Industrie (BDI) (2020), Acht-Punkte-Steuerplan zur Bewältigung der Corona-Krise, Stellungnahme, 26. März, https://bdi.eu/media/publikationen/\#/publikation/news/acht-punkte-steuerplan-zur-bewaeltigung-der-corona-krise/ (23. April 2020).

Djankov, S., T. Ganser, C. McLiesh, R. Ramalho und A. Shleifer (2010), The Effect of Corporate Taxes on Investment and Entrepreneurship, American Economic Journal: Macroeconomics, 2(3), 31-64.

Dobbins, L. und M. Jacob (2016), Do corporate tax cuts increase investments?, Accounting and Business Research, 46(7), 731-759.

Eberhartinger, E. und D. Langenmayr (2020), Coronakrise: Der steuerpolitische Handlungsbedarf groß, Die Presse, 20. April, https://www.diepresse.com/5801555/coronakrise-der-steuerpolitische-handlungsbedarfgross (23. April 2020).

Eberhartinger, E. und M. Petutschnig (o.D.), Steuerliche Maßnahmen zur Verbesserung der Liquidität der Unternehmen in der Corona Krise: Drei Vorschläge, https://www.wu.ac.at/fileadmin/wu/d/i/steuerlehre/Aktuelles/Corona_3Vorschläge.pdf (23. April 2020).

Gassen, J., M. Jacob, R. Maiterth, J. Müller, K. Nicolay, R. Ortmann, D. Schanz, C. Sureth-Sloane und J. Voget (2020), Verlustrücktrag zur Bekämpfung von Liquiditätsengpässen, https://accounting-for-transparency.de/wp-content/uploads/2020/03/trr266_verlustrücktrag_coronasoforthilfe.pdf (23. April 2020).

Giese, H., B. GraßI, S. Holtmann und P. Krug (2020), Steuerliche Entlastungsmöglichkeiten für kleine und mittlere Unternehmen wegen der COVID19-Pandemie - Kann das Steuersystem Solidarität in der Gesellschaft fördern?, Das deutsche Steuerrecht (DStR), 58, 752-760.

Graham, J. (1996), Debt and the Marginal Tax Rate, Journal of Financial Economics, 41(1), 41-74.

Graham, J. und H. Kim (2009), Sulating Corporate Marginal In-come Tax Rates and Implications for Corporate Debt Policy, Working Paper: Duke University and NBER.

Koch, R. und D. Langenmayr (2020), Corona-Krise: Steuerliche Belastungen für Unternehmen in Verlustsituationen reduzieren, https://www.ku.de/ wwf/forschung/forschungsinstitute/ku-research-institute-for-taxation/ oeffentlichkeitsarbeit/wortmeldungen/corona-krise/ (23. April 2020).

Langenmayr, D. und R. Lester (2018), Taxation and Corporate Risk-Taking, The Accounting Review, 93(3), 237-266.

Ökonomenpanel von ifo und FAZ (2020), Belastung durch Unternehmensteuern in Deutschland senken - ist das der richtige Weg?, 17. März, https://www.ifo.de/node/53691 (23. April 2020).

Oestreicher, A., R. Koch, D. Vorndamme und S. Hohls (2012), Aufkommenswirkungen einer Abschaffung des Ergebnisabführungsvertrags bei der ertragsteuerlichen Organschaft, IFSt-Schrift, 482.

Oestreicher, A., R. Koch, D. Vorndamme und S. Hohls (2013), ASSERT - Assessing the effects of reforms in taxation - a microsimulation approach, FAT Working Paper, 14-001.

Ohrn, E. (2018), The Effect of Corporate Taxation on Investment and Financial Policy: Evidence from the DPAD, American Economic Journal: Economic Policy, 10(2), 272-301.

Sachverständigenrat zur Begutachtung der gesamtwirtschaftlichen Entwicklung (SVR für Wirtschaft) (2020), Sondergutachten 2020: Die gesamtwirtschaftliche Lage angesichts der Corona-Pandemie, https://www.sachverstaendigenrat-wirtschaft.de/sondergutachten-2020.html (23. April 2020).

Spengel, C., F. Schmidt, J. Heckemeyer und K. Nicolay (2018), Effective Tax Levels Using The Devereux/Griffith Methodology, https://ec.europa.eu/taxation_customs/sites/taxation/files/final_report_2018_effective_tax_levels_revised_en.pdf (23. April 2020).

Statistisches Bundesamt (2020), Körperschaftsteuerstatistik 2015, Fachserie 14, Reihe 7.2, 28. Februar.

Zwick, E. und J. Mahon (2017), Tax Policy and Heterogeneous Investment Behavior, American Economic Review, 107(1), 217-248.

Title: The Tax Treatment of Losses: Options for Reforms During the Corona Crisis

Abstract: Due to the corona pandemic, many companies will suffer losses in 2020. The German tax system treats profits and losses asymmetrically: Whereas profits are taxed immediately, losses do not generate immediate tax refunds. This article discusses different options to improve the tax treatment of companies in loss situations. A microsimulation of these reforms reveals that extending the maximum amount for loss carry-back has a larger impact than extending the loss carry-back period. Enhancing loss carry-back opportunities also creates strong investment incentives. JEL Classification: $H 12, H 25$, E37 